\title{
Stereotactic radiosurgery for the treatment of stage I non-small cell lung cancer in high-risk patients
}

\author{
Arjun Pennathur, MD, ${ }^{\mathrm{a}}$ James D. Luketich, MD, ${ }^{\mathrm{a}}$ Dwight E. Heron, MD, ${ }^{\mathrm{b}}$ Ghulam Abbas, MD, ${ }^{\mathrm{b}}$ Steven Burton, MD, \\ Mang Chen, MD, ${ }^{a}$ William E. Gooding, MS, ${ }^{\mathrm{c}}$ Cihat Ozhasoglu, PhD, ${ }^{\mathrm{b}}$ Rodney J. Landreneau, MD, ${ }^{\mathrm{a}}$ and Neil A. Christie, MD ${ }^{\mathrm{a}}$
}

Objective: Surgical resection is the standard of care for patients with stage I non-small cell lung cancer. For highrisk patients, however, stereotactic radiosurgery may offer an alternative. We report our initial experience with stereotactic radiosurgery for treatment of stage I non-small cell lung cancer by a team of thoracic surgeons and radiation oncologists.

Methods: Patients medically ineligible for operation were offered stereotactic radiosurgery. Thoracic surgeons evaluated all patients, placed fiducials, and performed treatment planning in collaboration with radiation oncologists. Median dose of $20 \mathrm{~Gy}$ to $80 \%$ isodose line was administered as single fraction (range 20-60 Gy,1-3 fractions). Initial response rate, progression, and survival were monitored.

Results: Twenty-one patients underwent stereotactic radiosurgery in 3 years. Fiducial placement resulted in pneumothorax requiring a pigtail catheter in 10 of 21 patients $(47 \%)$. Disease showed initial response in 12 of 21 patients $(57 \%)$, was stable in $5(24 \%)$, progressed in $3(14 \%)$, and was not evaluable in $1(5 \%)$. Procedure-related mortality was zero. With mean 24-month follow-up, estimated 1-year survival probability was $81 \%$ (68\% confidence interval 0.73-0.90). Median survival was 26.4 months (confidence interval 19.6 months-not reached). Local progression occurred in 9 patients $(42 \%)$. Median time to local progression was 12.3 months (confidence interval 12 months-not reached).

Conclusion: Preliminary experience indicates that stereotactic radiosurgery (median dose $20 \mathrm{~Gy}$ ) is safe in this high-risk group; however, it was associated with significant local progression. Further prospective studies with multiple fractions are needed to evaluate its efficacy in this population.

Lung cancer is the most common cause of cancer-related mortality in the United States. Surgical resection is the standard treatment for stage I non-small cell lung cancer (NSCLC). ${ }^{1-3}$ With an aging population, many patients with otherwise resectable lung cancer have other comorbidities, which may preclude surgical resection. ${ }^{4}$ For these patients, conventional external beam radiotherapy is typically offered as treatment, with reported 5-year survivals of $10 \%$ to $30 \% .^{5-8}$ These results of conventional external beam radiation have not been satisfactory, prompting investigators to study other modalities of treatment for this highrisk group of patients with lung cancer.

Higher doses of radiation increase local control and cancer-specific survival. ${ }^{6}$ Increasing the dose with conventional radiation therapy techniques also increases the dose to normal tissue, however, resulting in increased toxicity. Stereo-

\footnotetext{
From Heart, Lung, and Esophageal Surgery Institute, ${ }^{\mathrm{a}}$ and Department of Radiation Oncology, ${ }^{\mathrm{b}}$ University of Pittsburgh Medical Center, and The University of Pittsburgh Cancer Institute Biostatistics Facility, ${ }^{\mathrm{c}}$ Pittsburgh, Pa.

Read at the Eighty-eighth Annual Meeting of The American Association for Thoracic Surgery, Washington, DC, May 5-9, 2007.

Received for publication May 8, 2007; revisions received April 21, 2008; accepted for publication June 15, 2008.

Address for reprints: James D. Luketich, MD, Henry T Bahnson Professor of Cardiothoracic Surgery, Chief, The Heart, Lung, and Esophageal Surgery Institute, University of Pittsburgh Medical Center, 200 Lothrop St; C-800, Pittsburgh PA 15213 (E-mail: luketichjd@upmc.edu).

J Thorac Cardiovasc Surg 2009;137:597-604

$0022-5223 / \$ 36.00$

Copyright (C) 2009 by The American Association for Thoracic Surgery

doi:10.1016/j.jtcvs.2008.06.046
}

tactic radiosurgery (SRS), a term coined by Leksell, is an approach that combines multiple convergent beams, precise localization with a stereotactic coordinate system, rigid immobilization, and single-fraction treatment. ${ }^{9}$ SRS is well established for the treatment of intracranial malignancies, and its use in extracranial malignancies is now being explored. In 1994, Lax and colleagues ${ }^{10}$ from the Karolinska Institute described a technique of extracranial SRS with a custom-made body cast and stereotactic coordinates. ${ }^{10}$ There have been few reports from the United States of application of SRS to the treatment of lung malignancies This modality of treatment is evolving. ${ }^{11-15} \mathrm{We}$ have previously reported on our experience with SRS in patients with primary and metastatic lung neoplasms. ${ }^{15}$ In this article, we present our preliminary results with the use of SRS for the treatment of stage I NSCLC in medically inoperable patients.

\section{MATERIALS AND METHODS}

We reviewed retrospectively our experience at the University of Pittsburgh during a 3-year period from 2002 to 2005 with SRS for the treatment of stage I NSCLC in patients who cold not undergo surgery. Informed consent was obtained from all patients, and the study was approved by the institutional review board at the University of Pittsburgh.

\section{Selection of Patients}

Patients with NSCLC routinely underwent staging with chest computed tomographic (CT) scan $(21 / 21,100 \%)$. Most patients $(18 / 21,86 \%)$ also underwent a positron emission tomographic (PET) scan. The patient characteristics are summarized in Table 1. Patients with mediastinal lymph nodes longer than $1 \mathrm{~cm}$ in short axis or a positive PET scan underwent 


\section{Abbreviations and Acronyms \\ $\mathrm{CT}=$ computed tomographic \\ $\mathrm{FEV}_{1} \quad=$ forced expiratory volume in 1 second \\ NSCLC $=$ non-small cell lung cancer \\ PET = positron emission tomographic \\ RECIST $=$ Response Evaluation Criteria in Solid Tumors \\ SRS $=$ stereotactic radiosurgery}

mediastinoscopy. Mediastinoscopy was performed in 2 patients. The inclusion criteria for SRS in the treatment of patients with stage I NSCLC were as follows: (1) patients who were considered to have medically inoperable disease because of poor pulmonary function (predicted postoperative forced expiratory volume in 1 second $\left[\mathrm{FEV}_{1}\right]$ less than $40 \%$ or diffusion capacity of the lung for carbon monoxide of less than $40 \%$ ), high cardiac risk, or other associated comorbidities, or (2) patient refusal for surgery. In this series, all patients had medically inoperable disease. All patients were evaluated by a thoracic surgeon and a radiation oncologist before treatment. Treatment was delivered with the CyberKnife (Accuray, Sunnyvale, Calif) system, a frameless system that consists of a 6-MV linear accelerator mounted on a computer-controlled robotic arm. The technology and the protocol used with this system have previously been described in detail. ${ }^{11,15}$

\section{Treatment Protocol}

A total of 1 to 4 fiducials, which are small tumor markers, were placed percutaneously with CT guidance by thoracic surgeons in and around the tumor for tumor tracking. An immobilization device (Alpha Cradle; Smithers Medical Products, North Canton, Ohio), which partially immobilizes the patient to decrease the motion and provide a reproducible setup, was custom made for each patient. A week to 10 days after placement of fiducials, a contrast-enhanced CT scan of the chest and upper abdomen was performed. The thoracic surgeon and radiation oncologist then evaluated the CT scan and jointly formulated a treatment plan. We made treatment volume considerations to avoid radiation injury in the surrounding normal lung, spinal cord, heart, aorta, liver, and stomach. We used the treatment planning software to outline the gross tumor volume. In addition, we sought to add a 5- to 10-mm radial margin to the treated volume, although this goal was limited by the opposing goal of avoiding toxicity to adjacent critical structures. On the day of the treatment, the patient was repositioned accurately to simulate the original planning setup. Tumor motion resulting from patient respiration was minimized by two techniques. A breath-holding method was used in 16 patients, whereas a dynamic tumor-tracking system (Synchrony; Accuray) was used in the remaining 5 patients. Targeting was coupled with a realtime image-guidance system. Patients were treated with 20 Gy prescribed to the $80 \%$ isodose line (1-3 fractions, total dose $20-60 \mathrm{~Gy}$ ). The biologically effective dose of $20 \mathrm{~Gy}$, determined by the linear quadratic equation, is estimated to be equivalent to about 60 to $70 \mathrm{~Gy}$. Early in our experience, we used a 20-Gy dose; as we gained more experience, we increased this to a total of $60 \mathrm{~Gy}$ for peripheral lesions. This change was made with caution because of the reported toxicity associated with an increased dose. A dose of $20 \mathrm{~Gy}$ in a single fraction was administered in 17 patients; the last 4 patients with peripheral lesions were treated with a total dose of $60 \mathrm{~Gy}$ in three fractions.

\section{Follow-up of Patients and Assessment of Response}

Patients were followed up at 3-month intervals with clinical examinations, CT scans, and PET scans. Modified Response Evaluation Criteria in Solid Tumors (RECIST) criteria, incorporating not only lesion size but also the quality of the lesion and metabolic uptake on PET scan, were used to assess response to treatment at 3 months ${ }^{15-17}$ (Table 2). Patients
TABLE 1. Patient characteristics

\begin{tabular}{lr}
\hline Total patients (No.) & 21 \\
Sex (male/female) & $9: 12$ \\
Age (y) & 71 \\
Median & $61-85$ \\
Range & \\
Stage (No.) & 14 \\
IA & 7 \\
IB & 21 \\
Staging procedures* (No.) & 18 \\
Computed tomographic scan & 2 \\
Positron emission tomographic scan & 8 \\
Mediastinoscopy & 6 \\
Histologic type (No.) & 6 \\
Squamous & 1 \\
Adenocarcinoma & \\
Non-small cell lung cancer (unspecified) & 6 \\
Not determined & 14 \\
Reason for stereotactic radiosurgery $\dagger$ (No.) & 6 \\
Poor pulmonary function test results & \\
Increased cardiac risk & \\
Multiple comorbidities & \\
*Most patients underwent multiple staging procedures. $\dagger$ Some patients had multiple \\
reasons for stereotactic radiosurgery. \\
\end{tabular}

were evaluated for initial response rate, time to progression, and overall survival.

\section{Data Collection and Statistical Analysis}

The objective of the study was to determine the outcomes of SRS in the treatment of stage I NSCLC. Information on patient demographic characteristics, tumor characteristics, treatment, and comorbidities (Charlson comorbidity index) was collected. ${ }^{18}$ Specific end points studied were complications, clinical response rates, time to progression, and overall survival. The pretreatment CT scan was used as a baseline for evaluation of response and disease progression. Local disease progression of the treated lesion was assessed in accordance with the modified RECIST criteria relative to baseline diameter. The time to progression was calculated from the treatment date after censoring data from patients who died without progression. Kaplan-Meier plots were constructed with $68 \%$ Greenwood confidence limits. Association between categoric variables was tested with the Fisher exact test or the $\chi^{2}$ test.

\section{RESULTS}

A total of 21 patients underwent SRS during a 3-year period. There were 9 men and 12 women, with a median age of 71 years (range, $61-85$ years). The most common reason for SRS was poor pulmonary function test results precluding curative resection $(14 / 21,67 \%)$. In this group of patients with poor pulmonary function, the median $\mathrm{FEV}_{1}$ was $0.66 \mathrm{~L}$ (range, 0.5-0.86 L), median $\mathrm{FEV}_{1}$ was $25 \%$ (range, $15 \%-$ $46 \%$ ), and median lung diffusion capacity for carbon dioxide was $30.5 \%$ of predicted (range, $19 \%-58 \%$ ). Several patients had multiple comorbidities, and the mean Charlson comorbidity index was 5.2 (median 5 , range $0-10$ ). There were 14 patients with stage IA NSCLC and 7 with stage IB. The mean size of the lesion was $2.2 \mathrm{~cm}$ (range, $0.9-5.5 \mathrm{~cm}$ ). The patient characteristics are summarized in Table 1. 
TABLE 2. Modified Response Evaluation Criteria in Solid Tumors criteria

\begin{tabular}{|c|c|c|c|}
\hline Response & CT mass size & CT mass quality & PET* \\
\hline Complete (meet any 2 criteria) & $\begin{array}{l}\text { Lesion disappearance (scar) or } \\
\quad<25 \% \text { original size }\end{array}$ & Cyst cavity formation; low density & SUV $<2.5$ \\
\hline Partial (meet any 1 criterion) & $\begin{array}{l}\text { Decrease }>30 \% \text { in sum of LDs of } \\
\text { target lesions }\end{array}$ & $\begin{array}{c}\text { Mass central necrosis or central } \\
\text { cavity with liquid density }\end{array}$ & $\begin{array}{l}\text { Decreased SUV or FDG uptake } \\
\text { area }\end{array}$ \\
\hline $\begin{array}{l}\text { Stable lesion (meet any } 1 \\
\text { criterion) }\end{array}$ & $\begin{array}{l}\text { Decrease }<30 \% \text { in sum of LDs of } \\
\text { target lesions }\end{array}$ & $\begin{array}{l}\text { Mass solid appearance, no central } \\
\text { necrosis or cavity }\end{array}$ & $\begin{array}{l}\text { Unchanged SUV or FDG uptake } \\
\text { area }\end{array}$ \\
\hline Progression (meet any 2 criteria) & $\begin{array}{l}\text { Increase }>20 \% \text { in sum of LDs of } \\
\text { target lesions }\end{array}$ & $\begin{array}{l}\text { Solid mass, invasion of adjacent } \\
\text { structures }\end{array}$ & $\begin{array}{l}\text { Higher SUV or larger FDG uptake } \\
\text { area }\end{array}$ \\
\hline
\end{tabular}

$C T$, Computed tomographic; $P E T$, positron emission tomography; $S U V$, standardized uptake value (of fluorodeoxyglucose $\mathrm{F} 18$ ); $L D$, lesion diameter; $F D G$, fluorodeoxyglucose $\mathrm{F}$ 18. *Positron emission tomography was performed selectively.

\section{Complications}

After percutaneous fiducial placement, 10 patients $(10 / 21$, $47 \%$ ) had pneumothorax that necessitated placement of a pigtail catheter. The median hospital stay after placement of fiducials was 1 day (range 0-17 days). One patient was admitted with exacerbation of chronic obstructive pulmonary disease after SRS. There were no procedure-related deaths.

\section{Initial Response}

Response was assessed at 3 months according to the modified RECIST criteria, as described previously ${ }^{15-18}$ (Table 2). Response could not be evaluated in 1 patient because of early death from pneumonia at 3.6 months. In the remaining patients, an initial complete response was observed in 7 patients $(7 / 20,35 \%)$, partial response was observed in 5 $(5 / 20,25 \%)$, stable disease was observed in $5(5 / 20$, $25 \%$ ), and progression occurred in 3 patients $(3 / 20,15 \%)$.

\section{Survival}

There were no procedure-related deaths. There were 10 deaths during the follow-up period. The mean follow-up of the remaining patients was 24 months (median 21 months, range 12-43 months). The probability of overall survival at 1 year was estimated to be $81 \%$ (68\% confidence interval $73 \%-90 \%)$. The median survival was 26.4 months $(68 \%$ confidence interval 19.6 months-not reached; Figure 1). During follow-up, local progression occurred in 9 patients $(42 \%, 68 \%$ confidence interval $32 \%-54 \%)$, and the median time to local progression was 12.3 months $(68 \%$ confidence interval 12 months-not reached).

\section{DISCUSSION}

Surgery is the treatment of choice for stage I NSCLC and offers the best chance for cure. ${ }^{1-3}$ For patients with lung cancer who cannot be operated on for medical reasons, there are few effective curative options. The primary standard treatment for medically inoperable stage I NSCLC is conventional external beam radiation therapy alone. Many patients who cannot undergo operation are elderly, with multiple comorbidities, and are typically not treated with chemotherapy. ${ }^{19}$ Sibley and colleagues ${ }^{6}$ reported the results of radiation therapy in 141 patients with stage I NSCLC. The median survival was 18 months, and the estimated overall survival at 2 and 5 years was $39 \%$ and $13 \%$, respectively. Local failure alone represented the most common mode of failure $(42 \%)$. Krol and colleagues ${ }^{7}$ reported the results in 108 patients with medically inoperable stage I NSCLC. The overall survival was estimated to be $75 \%, 31 \%$, and $15 \%$ at 1,3 , and 5 years, respectively. Local progression occurred in 71 patients $(65.7 \%)$. Finally, Qiao and colleagues, ${ }^{8}$ in a meta-analysis of the results of radiation therapy for stage I NSCLC, reported estimated overall 3 - and 5-year survival of $34 \%$ and $21 \%$, respectively. Because the results of conventional external beam radiation are suboptimal, novel treatment approaches such as SRS and radiofrequency ablation (RFA) may be applicable in this high-risk group of patients. ${ }^{1-17}$ The results of SRS for the treatment of stage I NSCLC in our study compare favorably with the previously mentioned studies in which conventional radiotherapy was used.

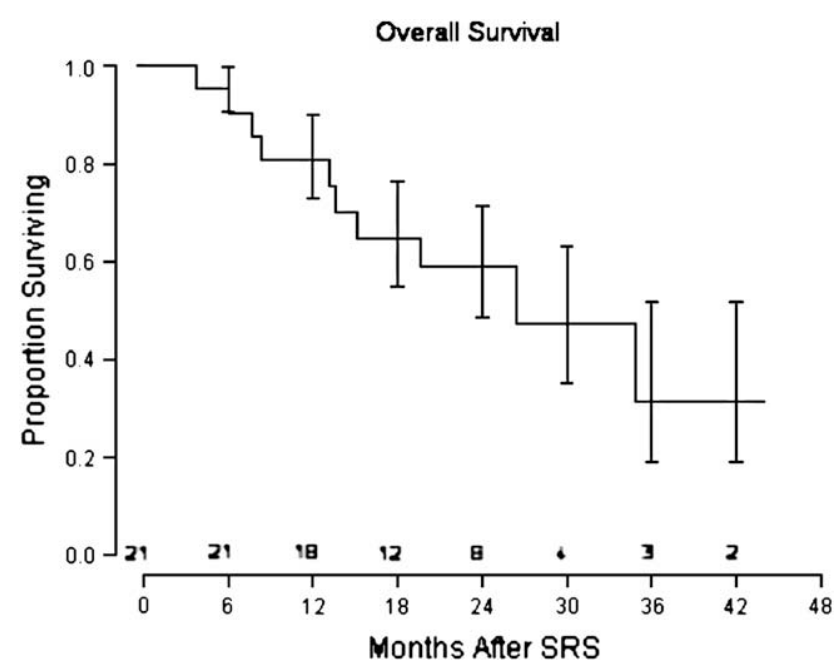

FIGURE 1. Kaplan-Meier plot illustrating overall survival for entire group. Time shown on $\mathrm{x}$-axis is in months from stereotactic radiosurgery (SRS). Error bars indicate $68 \%$ confidence intervals for probability of overall survival. 
The assessment of response is challenging after ablative therapies such as SRS and radiofrequency ablation (RFA), because many treated patients have residual scarring. We therefore used modified RECIST criteria that incorporated not only lesion size but also lesion quality and assessment of metabolic activity by PET scanning to evaluate response. ${ }^{15-17}$ There is, however, no consensus in the literature with regard to the determination of initial response, and ultimately recurrence and survival are the end points that need to be evaluated to assess the efficacy of treatment with such ablative therapies as SRS.

In this article, we report our initial experience in patients with stage I NSCLC treated with SRS using a frameless stereotactic system. Whyte and colleagues ${ }^{11}$ provided the first report from the United States with a similar system for frameless SRS in 23 patients treated with a single fraction of $15 \mathrm{~Gy}$. SRS has also been reported in several other parts of the world, including Japan, where an early study was performed by Uematsu and associates. ${ }^{20-25}$ Timmerman and coworkers $^{12}$ reported results for 37 patients with stage I NSCLC treated with SRS. In that dose escalation study, the dose was escalated from 24 Gy to $60 \mathrm{~Gy}$ in three fractions. At a median follow-up of 15 months, 6 patients had local failure; all had received less than 18 Gy per fraction. Local progression occurred between 3 and 18 months (median 13 months) after treatment. The disease-free and overall survivals at median follow-up of 15 months were $50 \%$ and $64 \%$, respectively.

The biologically effective dose is estimated to be much higher with SRS than with standard external beam radiation. Biologically effective dose and local control have been reported on by Onishi and colleagues. ${ }^{22}$ Onishi and colleagues ${ }^{22}$ evaluated clinical outcomes of 245 patients with stage I NSCLC from 13 Japanese institutions and reported a lower local recurrence rate when the biologically effective dose of more than $100 \mathrm{~Gy}$ was used. In our study, the estimated median biologically effective dose was about 60 to $70 \mathrm{~Gy}$, and a significant incidence of local progression was noted. In addition to the dose, other factors that may contribute to local progression include the margins obtained around the tumor and the technique of tumor tracking during respiration.

\section{Dose Escalation: Local Control and Toxicity}

Increase in the dose improves local control; however, it may also be associated with increased toxicity, particularly when treating central lesions. Timmerman and colleagues ${ }^{13}$ recently reported their results with SRS (60-66 Gy in three fractions) in a phase II study of 70 patients with medically inoperable stage I NSCLC. At a median follow-up of 17.5 months, Timmerman and colleagues ${ }^{13}$ reported good local control of $95 \%$ and a 2 -year overall survival of $54 \%$. There was excessive toxicity noted in central tumors, however, with a 2-year freedom from severe toxicity of $54 \%$ in central lesions versus $83 \%$ in peripheral lesions. Of the 28 deaths during follow-up, $6(8.6 \%, 6 / 70)$ were treatment related. This increase in procedure-related mortality and the increase in toxicity, particularly with central lesions, led Timmerman and colleagues ${ }^{13}$ to conclude that this regimen should not be used for patients with tumors near central airways.

Le and colleagues ${ }^{14}$ reported the results of a dose escalation study in patients with lung neoplasm with single-fraction treatment. There were 21 patients with stage I NSCLC and 11 with metastatic tumors. The doses administered were less than 20 Gy in 10 patients, 25 Gy in 20 , and 30 Gy in 2. At a median follow-up of 18 months, Le and colleagues ${ }^{14}$ reported a 1-year survival of $85 \%$ for patients with stage I NSCLC. The 1-year freedom from local progression was $91 \%$ for patients who received a dose greater than $20 \mathrm{~Gy}$, versus $54 \%$ for those who received less than 20 Gy. Late toxicity was observed in 8 patients, however, all of whom had received more than $20 \mathrm{~Gy}$. There were 3 deaths related to the procedure, for a treatment related mortality of $9.7 \%$. All the patients who died had received more than 25 Gy in a single fraction and had centrally located lesions. In addition, all these patients had received chemotherapy, and 2 of the 3 had received previous radiation. Some of these patients had received gemcitabine, which is associated with radiation-recall toxicity. These studies show that although local control with SRS may be very good with higher doses, caution must be exercised in selecting the dose schema, particularly for patients with central lesions.

In our study, in which a lower dose was used, no treatmentrelated deaths were observed. It is important, however, to note that some toxicity may occur late, and further follow-up is required to determine any late toxicity. Our current approach is to treat only peripheral lesions with a higher dose of $60 \mathrm{~Gy}$ in 3 fractions to balance the toxic effects with local control. Further, as highlighted by Le and colleagues, ${ }^{14}$ caution should be exercised, particularly with gemcitabine-based adjuvant therapy, when a large single fraction dose is used.

There are several radiosurgical treatment devices, and direct comparisons of results between these devices are not available. Some systems use the breath-holding technique and do not require placement of fiducials. ${ }^{12}$ Patients treated with such systems still require a CT-guided or bronchoscopic biopsy of the lesion, however, which may be complicated by pneumothorax. Other systems use a dynamic tracking system. In our center, we used the CyberKnife, which at present requires the placement of fiducials to help track tumor motion during respiration. One of the disadvantages of transthoracic placement of fiducials under CT guidance is the development of pneumothorax, particularly in these patients with medically inoperable disease because of pulmonary dysfunction. For these high-risk patients, we had a low threshold for placement of pigtail catheters to drain the pneumothorax. Recent advances in electromagnetic navigation-guided bronchoscopy and placement of fiducials may decrease the incidence of pneumothorax. ${ }^{26}$ 
One of the interesting aspects of this study is that thoracic surgeons were actively involved in patient selection, placement of fiducials, contouring the lesion, and treatment planning in close collaboration with radiation oncologists. The thoracic surgeons' expertise with regard to the anatomy, the physiologic assessment of the patient, and the ability to make an evaluation of the surgical risk was valuable in the selection of patients for SRS. It is particularly important that the determination of medical inoperability be made by the thoracic surgeon. One factor alone, such as poor pulmonary function test results, may not make a patient ineligible for operation. An interesting study demonstrated excellent survival after surgical resection in a highly selected group of patients with early-stage NSCLC and poor pulmonary function test results (mean $\mathrm{FEV}_{1}$ 0.7, $29 \%$ of predicted). ${ }^{27}$ In that study, after surgical resection with or without associated lung volume reduction surgery, the overall survival at 5 years was $68 \%$. The study emphasizes that one factor alone does not make a patient inoperable, and this decision regarding operability should be made by the thoracic surgeon after a comprehensive evaluation of the patient that includes not only pulmonary function tests but also other patient-specific factors and comorbidities.

The results of SRS in our study appear to be equivalent or superior to the reported results of conventional radiotherapy. ${ }^{5-8}$ Further prospective studies are required, however, for definitive comparison of SRS with conventional external beam radiation treatment or other emerging technologies such as RFA. Our study has the limitations inherent to retrospective studies, such as selection bias. In addition, longer follow-up for this cohort is needed, and full evaluation of survival end points will require greater maturity of time-to-event data. In this preliminary study, we used a median dose of 20 Gy in a single fraction in most cases, and minimal toxicity was observed. A significant incidence of local progression was noted. We now have an ongoing, prospective, institutional review board-approved study to evaluate the efficacy of a regimen with $60 \mathrm{~Gy}$ in three fractions for lesions that are not central in location in medically inoperable patients.

\section{CONCLUSIONS}

In summary, this study is a preliminary report on the use of SRS for the treatment of medically inoperable stage I NSCLC, and our results appear to be equivalent or superior to the results of conventional radiation therapy. Several factors merit further investigation, including optimal patient selection, appropriate dose, fractionation, and balancing the efficacy of the treatment against toxicity. In addition, the role of adjuvant therapy along with SRS remains to be determined. Surgical resection remains the standard treatment for stage I NSCLC in operable cases ${ }^{1-3}$; however, SRS may have a role for patients with medically inoperable disease. Prospective studies are currently in progress at our institution and others to address these issues and to define the role of SRS in the treatment of lung neoplasm. Thoracic surgeons should continue actively to investigate new technologies such as SRS and to add these techniques to their armamentarium in the treatment of lung neoplasm. In conclusion, SRS may provide an alternative option for high-risk patients with stage I NSCLC who are not medically fit for surgery.

\section{References}

1. Ginsberg RJ, Martini N. Non-small cell lung cancer: surgical management. In: Pearson FG, Cooper JD, Deslauriers J, Ginsberg RJ, Hiebert CA, Patterson GA, eds. Thoracic surgery. 2nd ed. Philadelphia: Churchill Livingstone; 2002:837-59.

2. Ginsberg RJ, Rubinstein LV, Lung Cancer Study Group. Randomized trial of lobectomy versus limited resection for T1 N0 non-small cell lung cancer. Ann Thorac Surg. 1995;60:615-23.

3. Landreneau RJ, Sugarbaker DJ, Mack MJ, Hazelrigg SR, Luketich JD, Fetterman L, et al. Wedge resection versus lobectomy for stage I (T1 N0 M0) non-small-cell lung cancer. J Thorac Cardiovasc Surg. 1997;113:691-700.

4. Loran DB, Zwischenberger JB. Thoracic surgery in the elderly. J Am Coll Surg. 2004; 199:773-84.

5. Jeremic B, Classen J, Bamberg M. Radiotherapy alone in technically inoperable, medically inoperable, early stage (I/II) non-small cell lung cancer. Int J Radiat Oncol Biol Phys. 2002;54:119-30.

6. Sibley G, Jamieson T, Marks L, Anscher MS, Prosnitz LR. Radiotherapy alone for medically inoperable stage I non-small-cell lung cancer: the Duke experience. Int J Radiat Oncol Biol Phys. 1998;40:149-54.

7. Krol AD, Aussems P, Noordijk EM, Hermans J, Leer JW. Local irradiation alone for peripheral stage I lung cancer: could we omit the elective regional nodal irradiation? Int J Radiat Oncol Biol Phys. 1996;34:297-302.

8. Qiao X, Tullgren O, Lax I, Sirzén F, Lewensohn R. The role of radiotherapy in treatment of stage I non-small cell lung cancer. Lung Cancer. 2003;41:1-11.

9. Song D, Kavanagh B, Benedict SH, Schefter T. Stereotactic body radiation therapy. Rationale, techniques, applications, and optimization. Oncology (Williston Park). 2004;18:1419-30; discussion 1430, 1432, 1435-6.

10. Lax I, Blomgren H, Näslund I, Svanström R. Stereotactic radiotherapy of malignancies in the abdomen: methodological aspects. Acta Oncol. 1994;33:677-83.

11. Whyte RI, Crownover R, Murphy MJ, Martin DP, Rice TW, DeCamp MM Jr, et al. Stereotactic radiosurgery for lung tumors: preliminary report of a phase I trial. Ann Thorac Surg. 2003;75:1097-101.

12. Timmerman R, Papiez L, McGarry R, Likes L, DesRosiers C, Frost S, et al. Extracranial stereotactic radioablation: results of a phase I study in medically inoperable stage I non-small cell lung cancer. Chest. 2003;124:1946-55.

13. Timmerman R, McGarry R, Yiannoutsos C. Excessive toxicity when treating central tumors in a phase II study of stereotactic body radiation therapy for medically inoperable early-stage lung cancer. J Clin Oncol. 2006;24:4833-9.

14. Le QT, Loo BW, Ho A, Cotrutz C, Koong AC, Wakelee H, et al. Results of a phase I dose-escalation study using single-fraction stereotactic radiosurgery for lung tumors. J Thorac Oncol. 2006;1:802-9.

15. Pennathur A, Luketich JD, Burton S, Abbas G, Heron DE, Fernando HC, et al Stereotactic radiosurgery for the treatment of lung neoplasm: initial experience. Ann Thorac Surg. 2007;83:1820-5.

16. Herrera LJ, Fernando HC, Perry Y, Gooding WE, Buenaventura PO, Christie NA, et al. Radiofrequency ablation of pulmonary malignant tumors in nonsurgical candidates. J Thorac Cardiovasc Surg. 2003;125:929-37.

17. Fernando HC, De Hoyos A, Landreneau RJ, Gilbert S, Gooding WE, Buenaventura PO, et al. Radiofrequency ablation for the treatment of non-small cell lung cancer in marginal surgical candidates. J Thorac Cardiovasc Surg. 2005;129:639-44.

18. Charlson ME, Pompei P, Ales KL, MacKenzie CR. A new method of classifying prognostic comorbidity in longitudinal studies: development and validation. $J$ Chronic Dis. 1987;40:373-83.

19. Grasse EL, Curran WJ Jr. Inoperable localized stage I and stage II non-small-cell lung cancer. Curr Treat Options Oncol. 2002;3:75-83.

20. Wulf J, Haedinger U, Oppitz U, Thiele W, Mueller G, Flentje M. Stereotactic radiotherapy for primary lung cancer and pulmonary metastases: a noninvasive treatment approach in medically inoperable patients. Int J Radiat Oncol Biol Phys. 2004;60:186-96.

21. Uematsu M, Shioda A, Tahara K, Fukui T, Yamamoto F, Tsumatori G, et al. Focal, high dose, and fractionated modified stereotactic radiation therapy for lung carcinoma patients a preliminary experience. Cancer. 1998;82:1062-70.

22. Onishi H, Araki T, Shirato H, Nagata Y, Hiraoka M, Gomi K, et al. Stereotactic hypofractionated high-dose irradiation for stage I nonsmall cell lung carcinoma: 
clinical outcomes in 245 subjects in a Japanese multiinstitutional study. Cancer. 2004;101:1623-31.

23. Zimmermann FB, Geinitz H, Schill S, Grosu A, Schratzenstaller U, Molls M, et al. Stereotactic hypofractionated radiation therapy for stage I non-small cell lung cancer. Lung Cancer. 2005;48:107-14.

24. Nyman J, Johansson KA, Hulten U. Stereotactic hypofractionated radiotherapy for stage I non-small cell lung cancer-mature results for medically inoperable patients. Lung Cancer. 2006;51:97-103.

25. Fritz P, Kraus HJ, Mühlnickel W, Hammer U, Dölken W, Engel-Riedel W, et al. Stereotactic, single-dose irradiation of stage I non-small cell lung cancer and lung metastases. Radiat Oncol. 2006;1:30.

26. Anantham D, Feller-Kopman D, Shanmugham LN, Berman SM, DeCamp MM, Gangadharan SP, et al. Electromagnetic navigation bronchoscopy-guided fiducial placement for robotic stereotactic radiosurgery of lung tumors: a feasibility study. Chest. 2007;132:930-5

27. Choong C, Meyers BF, Battafarano RJ, Guthrie TJ, Davis GE, Patterson GA, et al. Lung cancer resection combined with lung volume reduction in patients with severe emphysema. J Thorac Cardiovasc Surg. 2004;127:1323-31.

\section{Discussion}

Dr Jack A. Roth (Houston, Texas). Recent advances with 3-dimensional conformal techniques can compensate for lung motion, and they can allow delivery of very high single fractions to primary lung tumors in patients with clinical stage I lung cancer. The members of the Pittsburgh group are innovators in applying novel technologies to the treatment of thoracic cancers, and now they demonstrate the application of this technology to the treatment of lung cancer in patients with medically inoperable disease. Importantly, thoracic surgeons evaluated all patients and performed treatment planning in collaboration with radiation oncologists. As these novel technologies evolve, it is going to be critical for thoracic surgeons to continue to participate in the delivery of SRS and other new therapies.

There have been several series of patients with clinical stage I lung cancer treated with stereotactic radiation in the United States and Japan, and these include both medically inoperable and surgically resectable disease. Both the local control rates and the 5-year survivals in those studies appear to be comparable to those reported in surgical series. These studies administered considerably higher doses of radiation than used in this study, which could explain the high local failure rate that Pennathur and colleagues observed.

There are planned or completed phase II clinical trials, both in the United States under the Radiation Therapy Oncology Group and in Japan under the Japan Clinical Oncology Group, to evaluate SRS for the treatment of resectable stage I NSCLC. If these results are confirmed, this type of technology could be competitive with surgery for operable stage I disease, and I think that it is therefore imperative that we begin to consider the design of randomized clinical trials to eventually assess the benefit of SRS.

I have several questions. Dr Pennathur, one fraction of $20 \mathrm{~Gy}$, which has a biologic effective dose calculated by our radiation oncologists as $60 \mathrm{~Gy}$, is less than the conventional dose of $66 \mathrm{~Gy}$ in 33 fractions that's usually given to these patients. This could explain the high local recurrence rate. Why was such a low dose given, and are there plans to increase both the dose and the margins beyond those that you reported in your study?

Second, the patients had multiple comorbidities. Could you estimate the lung cancer-specific survival for your cohort of patients?

Finally, do the radiation oncologists at your institution plan to offer this technology at higher doses to patients with potentially operable disease?
Dr Pennathur. Thank you, Dr Roth, for your comments and questions. I'm going to take the third question first. No, our radiation oncologists are not going to offer SRS to patients with operable stage I NSCLC. We believe that the data is in evolution and although the data from Japan is interesting, do not yet support that. I will discuss further about this after addressing the first two questions.

In answer to your first question, yes, I think that the local recurrence rate in our series has been high. It's about $40 \%$ of local progression. I think several factors may have played a role in this. As you rightly pointed out, I think dose is the number one factor. A 20Gy dose has been estimated to be equivalent to anywhere between $60 \mathrm{~Gy}$ and $70 \mathrm{~Gy}$ and is estimated by the linear quadratic equation, which makes a lot of assumptions, so that the radiation oncologist who actually calculates it comes with a specific number. Everybody agrees that this is not very precise, but our estimate is that it's somewhere around $70 \mathrm{~Gy}$ or so. The second issue is that with higher doses, the three-fraction schema of 20 Gy times 3, a 60 Gy dose, is supposed to be equal to a biologic effective dose of $180 \mathrm{~Gy}$. Now, Timmerman and colleagues ${ }^{13}$ have shown that with central lesions, this regimen has been highly toxic. The mortality from using this regimen has been close to $10 \%$, which is somewhat alarming, but most of these patients who died had central tumors. Of the 6 deaths they had, 4 were of patients with central tumor. In our series of 21 patients, 11 had central tumors and 10 had peripheral tumors. Early on, when we started out using SRS, we used the lower dose. The first reason for this was caution. The second reason was that the pulmonary function test results in these patients were quite poor, and the radiation oncologists had some concerns about giving a high dose. I must add that during the last year and a half, we have gone up to three fractions of $20 \mathrm{~Gy}$ for peripheral lesions. In this series, 4 patients received a total of $60 \mathrm{~Gy}$ for peripheral lesions, but the follow-up is not long enough as for the original 20 patients, and almost all the recurrences were in the first 20-Gy group, so the local progression rate is significant.

In terms of the margins, yes, we shoot for a margin of at least 5 $\mathrm{mm}$, but we presented data about a year ago suggesting that if the margins were less than $1 \mathrm{~cm}$ in wedge resections, our recurrence rates were much higher. So we want to go for about a $1 \mathrm{~cm}$ margin, but this is limited by the critical structures around the lesion and by the radiation exposure that these critical structures sustain. Unfortunately, many times it's not $1 \mathrm{~cm}$. It's closer to $5 \mathrm{~mm}$.

Finally, I think the issue about recurrence is the technique. Early on, we used the breath-hold technique. Now we are using more dynamic tracking wherein the patient's breathing is dynamically tracked by the camera and then the radiation is delivered in sync with the patient's breathing. In this series, again, only 5 patients were treated that way. The rest were treated with the breath-hold technique. I think that all this may explain the increase in terms of local progression. Finally, I think the issue is that our aggressive follow-up and imaging also contributed to early detection of recurrence.

Now, the second question was related to the dose, Dr Roth?

Dr Roth. Yes.

Dr Pennathur. With respect to the dose, again, we have gone up to $60 \mathrm{~Gy}$ for peripheral lesions. For central lesions, we are looking at one of the Japanese regimens, which is 12 Gy times 4, for a 48-Gy dose, which gives a biologically effective dose of more than $100 \mathrm{~Gy}$. 
With respect to the final issue about whether we can use SRS in operable cases, I think that the Japanese literature is quite encouraging. Unfortunately, I think that there is a disconnect between the survival figures quoted in the Japanese literature and the survival figures quoted in the United States. I think, for example, some of the Japanese surgical series have had approximately $90 \%$ to $100 \%$ 5 -year survivals, with survivals in the $80 \%$ range quoted after SRS I think in the United States we have not seen survivals in the $90 \%$ range for stage I lung cancer at 5 years. I wonder whether there is some biologic difference in terms of the patients we are treating, perhaps in the basic substrate of the patients themselves. I think it would be reasonable for patients with medically inoperable disease to compare the CyberKnife with some other therapy for medically inoperable cases. With respect to SRS for surgically operable cases, I think that we may need more data in the United States to pursue this. Certainly, as you mentioned, this is a hotly investigated area, with a lot of interest from a lot of groups, including ours, and we are looking at this very closely. Thank you for your comments and excellent questions.

Dr Tomasz Grodzki (Szczecin, Poland). I congratulate you on your excellent presentation; however, I am still doubtful whether those patients really had inoperable disease, because if you evaluated them, you probably performed mediastinoscopy. This means that they survived anesthesia. If you placed those metal markers, it means that they were subjected to pneumothorax, and they all survived it. This was a kind of functional test for segmentectomy. I think I would prefer to perform segmentectomy and then radiation.

What was the N status of those patients? You didn't mention it. Was this clinical stage I or pathologic stage I?

Dr Pennathur. These patients, if they had enlarged lymph nodes and positive PET scan results in the mediastinum, underwent a mediastinoscopy and N2 disease was ruled out. Otherwise, they did not undergo any other invasive staging, such as video-assisted thoracoscopic surgical staging, for example. Two patients underwent mediastinoscopy, and they certainly did not have any invasive procedures before SRS. So this is primarily clinical staging, with invasive staging in selected patients, to rule out N2 disease. The issue of medical inoperability is a critical one, and I think that it is important that surgeons be involved, because what is medically inoperable for a thoracic surgeon is not the same as what is medically inoperable for a pulmonologist and is different still for a radiation oncologist. These patients had a low $\mathrm{FEV}_{1}$ of 0.6 and had multiple comorbidities. The median Charlson Comorbidity Index value was 5.5, and we have validated data suggesting that the incidence of postoperative complications is significantly higher on multivariate analysis once this score reaches 3 to 4 . So I do think that this was a highrisk group of patients. I think, however, that your point is excellent. There may be patients with predominant upper lobe emphysema and lung cancer there with low $\mathrm{FEV}_{1}$ who would certainly benefit from lung resection along with lung volume reduction. I therefore think that it's critical for thoracic surgeons to evaluate these patients before declaring the disease inoperable, and these patients were all very carefully assessed before they were subjected to SRS.

Dr Jessica S. Donington (Stanford, California). I congratulate you on this work. At Stanford we also feel that it's important for surgeons to embrace this kind of technology. We are really the experts on the treatment of early-stage lung cancer, and we need to know all the options that are available for our patients.
I have two questions. One has to do with the local recurrences. In our work, we've noticed a difference in local recurrences between $\mathrm{T} 1$ and T2 tumors. The ability to give the full isodose for those smaller tumors is much easier. Did you note this? Also, one of the significant differences we find between surgery and radiotherapy is clearly the treatment of the lymph nodes, and we did have more nodal recurrences than we would like. That was an area where there were a lot of recurrences. Can you comment on the difference between your local recurrences, nodal recurrences, and distant recurrences in these patients?

Dr Pennathur. We didn't really find a difference in terms of local progression-free survival between $\mathrm{T} 1$ and $\mathrm{T} 2$ tumors. Nine patients had local progression. In many instances, this is associated with regional and also distant disease, but it was counted as local progression. Local-only progression occurred in fewer than 5 patients. Sometimes people might report it that way, but for this study we decided that any local recurrences need to be considered. Thank you for your comments and questions.

Dr Mark Block (Hollywood, Florida). I enjoyed your presentation, and I congratulate your group on really providing objective leadership in studying the ablative therapies for these early lung cancers.

I have two questions. First, how do you deal with lesions that are close to important structures, like hilar lesions close to the pulmonary artery or the aorta, a lingular lesion that's next to a left ventricular free wall? You don't want to give them 70 Gy radiation.

Second, are you at all familiar with the new fiducial-free systems that are being marketed? The reason I have concerns about that is that in our local market, the person who owns the CyberKnife facility-because it's an independent facility-was boasting to me the other day about how they're going to get a fiducial-free system and then be able to take the patient right from the screening CT scanner to the CyberKnife to zap those little 5-mm lesions without ever having to worry about a biopsy. This leads me to the last point, which is that I think the use of this technology is unfortunately going to be driven more by marketing and promotion than by science. I think your group is in a unique position to provide the most objective data on evaluating this technology. Most of the other literature out there has been written by people with a financial interest in increasing SRS use. I therefore challenge you to think about ways in which you can influence the debate by more than simply presenting your objective data. There must be policy discussions about how we're going to manage these patients.

Dr Pennathur. Thank you, Dr Block. Those are really excellent thoughts. In terms of the fiducial-free system, we have heard about it and we have discussed it with the radiation oncologists and with the physicists. At this point, we are not going to get into it, because we don't have the data to prove that this fiducial less system is as accurate in tracking the tumor as the one we currently use with fiducials. Some tumors move about a good 2 or $3 \mathrm{~cm}$ with breathing, and whether the fiducial-free system is going to be tracking this, we really do not know. The issue in terms of the study of these newer technologies is that I think that thoracic surgeons should take a lead. We have been taking care of this for a long time, and we also look at things a little bit differently and more objectively in terms of recurrence and survival and so forth. I think that it will be critical for us to get involved with these newer technologies, and we are trying to evaluate every aspect critically and to identify patients who would benefit versus patients who might not benefit. 
With respect to your question about the hilar lesions, they tend to tolerate a 20-Gy dose, which equals a 70-Gy dose. The moment you go up to a higher dose, however, you add risk. I think in the Stanford series, a 25-Gy dose in a central location was associated with about 2 deaths, for single-fraction radiation. A 20-Gy dose, however, may be somewhat safer. The Japanese have proposed a newer schema, which is a total of $48 \mathrm{~Gy}$, with each fraction only about $12 \mathrm{~Gy}$ for central lesions. I think we do have to be a little careful about the central lesions and what kind of dose we need to use, and I think we need to follow, evaluate them, and study them more carefully.

Dr Todd L. Demmy (Buffalo, New York). I have two quick questions. Your group is also reporting on RFA for similar patients. How are you sorting out SRS technology versus the competing technology of RFA? By extension, we're finding patients with RFA sometimes don't have recurrence at the target lesion but at a location nearby, which points to a problem with this technology. You can only target what you can see on the initial CT scan, not the minimal residual disease that you can't image and that is presumably cleared better by lobectomy or segmentectomy. When you report your results, are you going differentiate the recurrences at the initial target area from lesions that recurred nearby in the downstream path of the tumor spread?

Dr Pennathur. Thanks for your comments, Dr Demmy. In terms of the RFA and CyberKnife, I think they can potentially be complementary. We absolutely don't use RFA for central lesions. We had 1 patient who underwent RFA and brachytherapy who had a fatal hemoptysis about 3 weeks after the procedure, and we do not know whether it was the brachytherapy or RFA which caused it, but we haven't used RFA since for any central lesions. We use a modified dose schema for the central lesions. In terms of peripheral lesions, they can be complementary. For example, if there is a recurrence after RFA, one could use SRS, and vice versa. And sometimes for a peripheral lesion, it is the choice of the physician as to the technology to use. I think the other point which you brought up, which is that we treat the small lesion which we see but we don't look into microscopic spread, is critical. I think the issue about margins becomes very important, and we believe that we need to have at least a $1-\mathrm{cm}$ margin. We evaluate our recurrences in the lobe as well as the progressed lesion, and I think the upcoming American College of Surgeons Oncology Group (ACOSOG) trial on RFA also is going to be reporting the same way. We thank the Association for the privelege of presenting this paper. 